\title{
A Sweet Presentation of a Bitter Disease: Acute Febrile Neutrophilic Dermatosis Associated with Coccidioidomycoses
}

\author{
Zainab A. Jafri, DO ${ }^{1}$, Lydia Shedlofsky, DO², Andrew Newman, DO², Travis Lam, DO², Yebabe \\ Mengesha, $\mathrm{MD}^{2}$ \\ ${ }^{1}$ Arizona College of Osteopathic Medicine, Glendale, AZ \\ ${ }^{2}$ Affiliated Dermatology and Dermatology Department at Honor Health, Scottsdale, AZ
}

\section{ABSTRACT}

Acute Febrile Neutrophilic Dermatosis, also known as Sweet Syndrome, is an uncommon inflammatory disorder. Though the exact etiology is unclear, it has been presented in association with various entities. The majority of cases present following upper respiratory infections or viral gastroenteritis. Other causes include drug-induced reactions, pregnancy-related manifestations, or in association with specific hematologic or solid tumors. Rarely, it has been associated with Coccidioidomycosis, a prevalent fungus endemic to the Southwestern regions of the United States with a literature review revealing only three previous cases of Coccidioidomycosis-associated Sweet Syndrome. Here we report two new cases in individuals residing in Arizona.

\section{INTRODUCTION}

Acute Febrile Neutrophilic Dermatosis (AFND) was first described by Robert Douglas Sweet in 1964 and thus eponymously termed Sweet Syndrome. ${ }^{1,2}$ AFND is subdivided into three categories based on clinical settings: classic, malignancy-associated, and drug-induced. ${ }^{3,4}$

Classic AFND is described in association with infections, inflammatory bowel disease, and pregnancy. Most commonly, classic AFND follows upper respiratory infection but has also been associated with Yersinia, Tuberculosis, Histoplasmosis, and Toxoplasmosis. Coccidioidomycosisassociated AFND has rarely been reported in literature.

AFND is an uncommon inflammatory condition characterized clinically by sudden onset of erythematous skin eruption, presenting as papules, nodules, and plaques commonly on the upper extremity, trunk, neck, and face. ${ }^{1}$ The histological hallmark is variably dense dermal infiltrate largely composed of mature neutrophils, together with edema of the papillary dermis. ${ }^{5}$

Coccidioidomycosis, also known as Valley Fever, is an airborne, fungal disease caused by Coccidioides immitis or Coccidioides posadasii, a soil-dwelling fungus prevalent in regions of Southwestern North and Central America. ${ }^{7}$ During 1998-2014, there were $\sim 147,000$ coccidioidomycosis cases reported to the Centers for Disease Control and Prevention (CDC) with, approximately two thirds occurring among Arizona residents. ${ }^{8}$ This typically presents as a subclinical pulmonary infection, with selfresolution in immunocompetent individuals. The skin along with lymph nodes, meninges, and the musculoskeletal system, are the

March 2021 Volume 5 Issue 2 
most common sites of disseminated infection. Cutaneous involvement generally presents as polymorphous lesions, resembling acne or rosacea.

Herein we examine two cases of AFND secondary to Coccidioidomycosis.

\section{CASE PRESENTATION}

\section{Case Report One}

A 55-year-old woman presented to her primary care physician with shortness of breath and rash. She was empirically treated with cefuroxime and amoxicillin without resolution in symptoms. Subsequently she presented to outpatient dermatology clinic a month later for evaluation of the rash. She presented with deep red, sharply demarcated nodules and papules that appeared edematous and vesiculated, some coalescing into larger groups, bilaterally on upper and lower extremities, back, chest and abdomen (Figure 1). No lesions appeared on the palms or soles. Two $4 \mathrm{~mm}$ punch biopsies were obtained from the thighs, and lab work was drawn. The biopsies returned with interstitial granulomatous dermatitis with papillary dermal edema and eosinophils, negative for fungal organisms, consistent with Histiocytoid Sweet Syndrome. Further workup resulted yielded positive serology for Coccidioidomycosis IgM and IgG antibodies. She was referred to the Infectious Disease team, who initiated treatment with oral fluconazole and oral corticosteroids. She continued to follow up, gradually tapering the oral steroids. At four months the rash had resolved leaving only post-inflammatory hyperpigmentation.

\section{Case Report Two}

A 57-year-old homeless gentleman presented to the Emergency Department

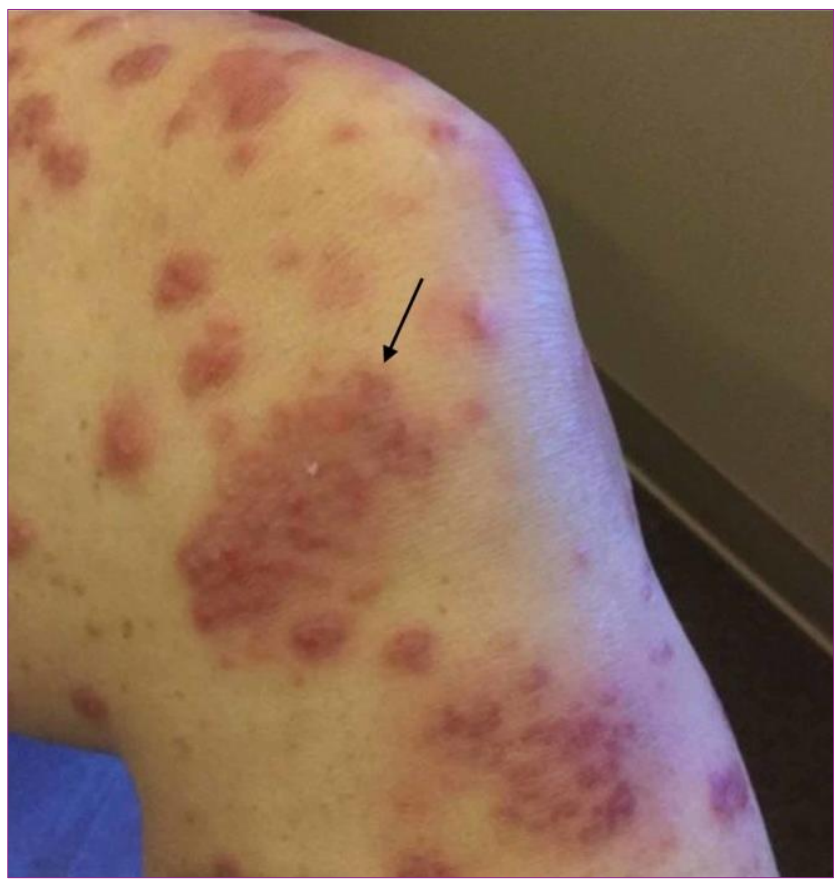

Figure 1. Left medial lower extremity revealing classic AFND architecture of erythematous plaques

complaining of a cough and a diffuse rash. He was initiated on ceftriaxone and azithromycin after being diagnosed with left lower lobe pneumonia. Review of systems was positive for headaches, fever, shaking chills, and nausea. The Dermatology service was consulted due to diffuse large, erythematous nodules coalescing into plaques on the left shoulder and neck (Figure 2). On physical examination firm nodules on the right forearm and chest were noted with several scattered vesicles on the extremities. Vesicles were swabbed for bacterial and viral cultures, with negative findings and several skin biopsies were obtained. Serology confirmed the presence of Coccidioidomycosis with a positive $\lg \mathrm{M}$. Biopsies revealed diffuse neutrophilic infiltrate with perivascular nuclear dust and massive papillary edema, all consistent with AFND. Infectious Disease and Pulmonology services were consulted, and the patient was initiated on intravenous corticosteroids with discussion of need for anti-fungal medications and careful monitoring. He 
gradually improved throughout his hospital stay but was lost to follow up upon discharge.

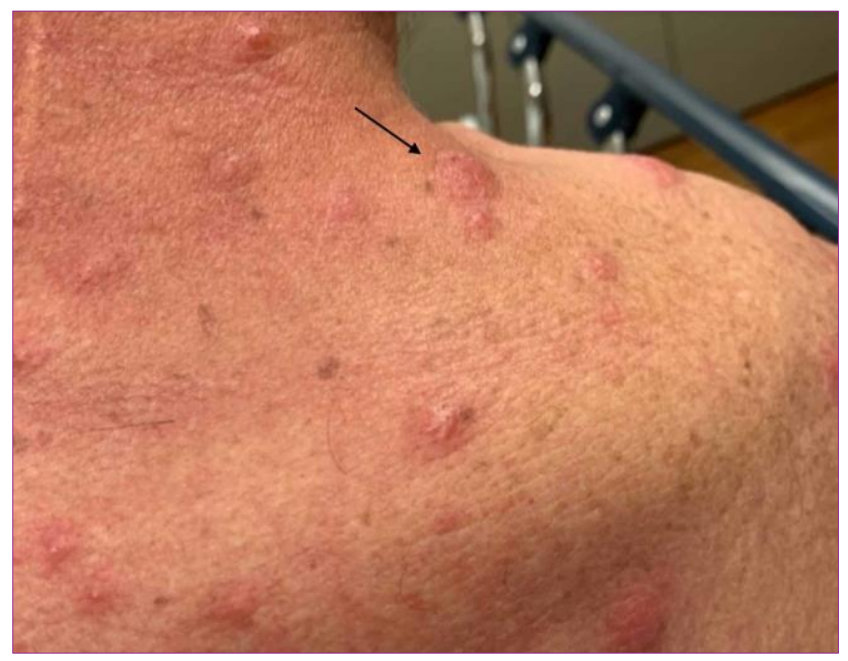

Figure 2. Posterior neck. Indurated erythematous nodules and pseudovesicles

\section{DISCUSSION}

The diagnosis of classic AFND requires the presence of both major criteria and two out of the four minor criteria (Table 1). ${ }^{4}$

\section{Symptoms and clinical manifestations typically respond rapidly to high-dose systemic corticosteroids. Second-line systemic agents include colchicine, dapsone, TNF-alpha antagonists and cyclosporine. . $^{4,6}$}

The pathogenesis for Acute Febrile Neutrophilic Dermatosis (AFND) is not fully elucidated but may be related to cytokinemediated recruitment of neutrophils to the skin. For this reason, AFND is treated firstline with corticosteroids, especially with extensive involvement, and has historically shown dramatic resolution. This disease has been associated with several clinical settings, and treatment should be guided by the underlying cause. Though rare, in endemic areas underlying fungal etiologies must be considered and diagnostic tests should be considered when index of suspicion is high, such as these two cases. Confirmatory testing for Coccidioidomycosis is needed for diagnosis. Diagnostic modalities such as serology, culture, and antibody screen are useful, as symptoms may be nonspecific.

Table 1. Sweet Syndrome Diagnostic Criteria

\begin{tabular}{|c|c|}
\hline & Criteria \\
\hline $\begin{array}{l}\text { Major } \\
\text { Criteria }\end{array}$ & $\begin{array}{l}\text { 1. Abrupt onset of painful erythematous } \\
\text { plaques or nodules } \\
\text { 2. Histopathological evidence of dense } \\
\text { neutrophil infiltrate in the dermis } \\
\text { without evidence of leukocytoclastic } \\
\text { vasculitis }\end{array}$ \\
\hline $\begin{array}{l}\text { Minor } \\
\text { Criteria }\end{array}$ & $\begin{array}{l}\text { 1. Fever }>38^{\circ} \mathrm{C} \\
\text { 2. Association with an underlying } \\
\text { hematological, or visceral } \\
\text { malignancy, inflammatory disease, or } \\
\text { pregnancy, or preceded by an upper } \\
\text { respiratory or gastrointestinal } \\
\text { infection or vaccination } \\
\text { 3. Response to treatment with } \\
\text { potassium iodide or steroids } \\
\text { 4. Abnormal laboratory values (3 of the } \\
\text { following } 4 \text { ) } \\
\text { a. Erythrocyte sedimentation } \\
\text { b. Posite greater than } 20 \mathrm{~mm} / \mathrm{h} \\
\text { c. }>8,000 \text { leukocytes } \\
\text { d. }>70 \% \text { neutrophils }\end{array}$ \\
\hline
\end{tabular}

Diagnosing the appropriate cause of AFND is critical in the treatment and management of the disease. General first-line therapy, corticosteroids, should be avoided as the sole treatment modality in infectious etiologies. ${ }^{9}$ Patients with AFND secondary to Coccidioidomycosis will typically require antifungal medication. Azole antifungals, specifically fluconazole and itraconazole are the initial therapy of choice for disseminated infections. ${ }^{7}$ Therefore, close monitoring and referral to Infectious Disease is recommended. In this setting consideration for optimization of therapy and management 
of the overall infection will improve prognosis and reduce patient morbidity.

These unique cases of Coccidioidomycosisassociated Sweet Syndrome demonstrate the importance of consideration of this uncommon etiology. Importance should be given to individuals residing in the endemic regions, such as Southwestern regions of the United States or with recent travel to these areas.

\section{CONCLUSION}

In conclusion, until now, three cases of Coccidioidomycosis-associated Acute Febrile Neutrophilic Dermatosis (AFND) have been reported. ${ }^{10}$ Coccidioides, fungi endemic to the Southwestern regions of the United States, Central, and South America is a common pathogen that must be considered in patients presenting with clinical signs of AFND. These patients should be followed with a treatment plan of antifungal therapy, in addition to systemic corticosteroids, to appropriately treat these rare cutaneous manifestations.

\section{Conflict of Interest Disclosures: None}

Funding: None

Corresponding Author:

Lydia B. Shedlofsky, DO

Affiliated Dermatology Honor Health

20401 N $73^{\text {rd }}$ Street \#230

Scottsdale, AZ 85255

Phone: 480-556-0446

Fax: 480-556-0447

Email: Ishedlofsky@affderm.com

\section{References:}

1. Korkut M. A dermatologic emergency; Sweet's syndrome. The American Journal of Emergency Medicine. 2019;37(9):1807.e1-1807.e3. doi:10.1016/j.ajem.2019.06.012

2. Sweet's syndrome (acute febrile neutrophilic dermatosis) - Journal of the American Academy of Dermatology. Accessed June 19, 2020. https://www.jaad.org/article/S01909622(94)70215-2/pdf

3. Nelson CA, Noe MH, McMahon CM, et al. Sweet syndrome in patients with and without malignancy: A retrospective analysis of 83 patients from a tertiary academic referral center. Journal of the American Academy of Dermatology. 2018;78(2):303-309.e4. doi:10.1016/j.jaad.2017.09.013

4. Cohen PR, Kurzrock R. Sweet's Syndrome. Am J Clin Dermatol. 2002;3(2):117-131. doi:10.2165/00128071-200203020-00005

5. Secchin P, Gomes MK, Quintella DC, et al. Idiopathic histiocytoid Sweet syndrome: a case report with clinical and histopathological considerations. Int J Dermatol. 2018;57(10):1182-1186. doi:10.1111/ijd.14159

6. Heath MS, Ortega-Loayza AG. Insights Into the Pathogenesis of Sweet's Syndrome. Front Immunol. 2019;10:414. doi:10.3389/fimmu.2019.00414

7. "The Acutely III Patient With Fever and Rash." Mandell, Douglas, and Bennett's Principles and Practice of Infectious Diseases, by John E. Bennett et al., 9th ed., Elsevier / Saunders, 2015, pp. 801-818.e4.

8. Jones JM, Koski L, Khan M, Brady S, Sunenshine R, Komatsu KK. Coccidioidomycosis: An underreported cause of death-Arizona, 2008-2013. Medical Mycology. 2018;56(2):172-179. doi:10.1093/mmy/myx041

9. Sweet's syndrome associated with myelodysplasia: possible role of cytokines in the pathogenesis of the disease - Reuss-Borst - 1993 - British Journal of Haematology - Wiley Online Library. Accessed June 19, 2020. https://onlinelibrary.wiley.com/doi/abs/10.1111/j.1 365-2141.1993.tb03083.x

10. DiCaudo DJ, Ortiz KJ, Mengden SJ, Lim KK. Sweet Syndrome (Acute Febrile Neutrophilic Dermatosis) Associated With Pulmonary Coccidioidomycosis. Arch Dermatol. 2005;141(7). doi:10.1001/archderm.141.7.881 